\title{
Editorial
}

\section{Kawasaki disease: reflections on pathological observations}

\author{
Anton E. Becker
}

I

$\mathrm{n}$ this issue Naoe and co-workers present an overview of the pathological changes in arteries in Kawasaki disease. ${ }^{1}$ The most important sequels of this disease relate to the occurrence of arteritis. The authors present a scheme of arterial changes (their Figure 14) which reveals that the inflammatory process from an acute stage onwards may either regress leading to full restoration or to a range of pathological stages. Once a panarteritis has developed, thrombosis and occlusion of the lumen is almost inevitable. At the same time, however, the weakened arterial wall may expand on the basis of local hemodynamics, causing an aneurysm to appear. Rupture may ensue under these circumstances. Indeed, one of the most common and often unexpected sudden causes of death is due to rupture of a coronary arterial aneurysm with cardiac tamponade as a direct consequence.

Does the inflammatory process provide a clue to etiology and pathogenesis?

The aforementioned pathological observations clearly indicate that the disease basically affects the arterial wall and has preference for middle-sized and smaller muscular arteries. The histopathology shows an acute stage characterized by influx of polymorphonuclear leukocytes, soon to be followed by an influx of lymphocytes and cells of the mononuclear phagocytic system. At that stage of the inflammatory process a granulomatous reaction is produced.

Although a granulomatous vasculitis is itself nondiagnostic with regard to etiology, it nevertheless introduces considerations with respect to pathogenesis. The concept being that macrophages are incapable of dissolving certain agents, which then remain present within the cytoplasm classical examples are lepromatous leprosy granulomas and those produced by the ingestion of silicon. The presence of aggressive substances within macrophages leads to a continuous stage of activation and, therefore, the ongoing production of inflammatory mediators such as cytokines. On the other hand, some form of a granulomatous inflammatory reaction show a delayed type allergic reaction, as in tuberculoid leprosy. In other words, auto-allergic processes should also be taken into consideration. The pathogenetic mechanisms that may underlay auto-allergic inflammatory diseases are manifold, but all are considered to relate directly to activation of the cellular autoimmune response normally present.

\section{What have we learned thus far?}

Although impressive knowledge has been accumulated over the years with respect to granulomatous inflammatory processes, it still remains enigmatic in most cases what causes the disease. Nevertheless, researchers should focus in particular on conditions that may lead to an incapacity of the macrophage to dissolve phagocytosed material and mechanisms of auto-allergy that may underlay the granulomatous reaction. From this point of view it is of major interest also that Kawasaki disease shows a distinct preference for certain parts of the world. The disease has been described first in Japan by Dr. Tomisaku Kawasaki ${ }^{2,3}$ and since then has been described in epidemic form in certain regions of the world, such as parts of the USA, but appears almost unheard of in other parts, such as large parts of Western Europe. ${ }^{4}$

These considerations also raise the question as to the relationship with another rare disease, known as the infantile form of periarteritis nodosa. It has been claimed that the histopathology of the arterial inflammatory process in this condition is very similar to that encountered in Kawasaki disease. ${ }^{5,6}$ Indeed, it has been suggested that both diseases are identical, the difference being that Kawasaki disease usually is diagnosed on the basis of clinical symptoms and signs, whereas infantile periarteritis nodosa usually is diagnosed on histological grounds. ${ }^{7}$ Such considerations may be helpful in finding new ways to understand pathogenesis and etiology of 
Kawasaki disease. Epidemiologists and basic scientists alike should focus on processes known to induce granulomatous inflammatory reactions, in perspective of epidemiological data. Combined efforts along these lines, preferably global wide, may eventually clarify the cause of Kawasaki disease.

\section{University of Amsterdam \\ Academic Medical Center \\ Meibergdreef 9 \\ 1105 AZ Amsterdam Zuidoost \\ The Netherlands}

\section{References}

1. Naoe S, Shibuya K, Takahashi K, Wakayama M, Masuda H, Tanaka N. On pathological observations concerning the cardiovascular lesions in Kawasaki disease. Cardiol Young 1991;
1: 212-220.

2. Kawasaki T. A new infantile acute febrile mucocutaneous lymph node syndrome. Jpn J Allergy 1967; 16: 178-222. [Japanese]

3. Kawasaki T, Kosaki F, Osawa S, Shigemarsu I, Yanagawa S. A new infantile acute febrile mucocutaneous lymph node syndrome (MLNS) prevailing in Japan. Pediatrics 1974; 54: 271 280.

4. Becker AE. Kawasaki disease: a pathology survey in Western Europe. Ped Pathol 1986; 6: 1-9.

5. Jones ME. Infantile polyarteritis nodosa: The expanded syndrome of Kawasaki-Fetterman. 2nd Joint Meeting Paediatric Pathology Club and Paediatric Pathology Society, Toronto, 1975.

6. Landing $\mathrm{BH}$, Larson EJ. Apparent identity of infantile periarteritis nodosa with coronary artery involvement (IPN) and mucocutaneous lymph node syndrome (MCLS): comparison of 20 patients from North America to patients from Hawaii and Japan. 2nd Joint Meeting Paediatric Parhology Club and Paediatric Pathology Society, Toronto, 1975.

7. Becker AE. Kawasaki disease. Lancet 1976; 1: 864. [Letter to the Editor] 